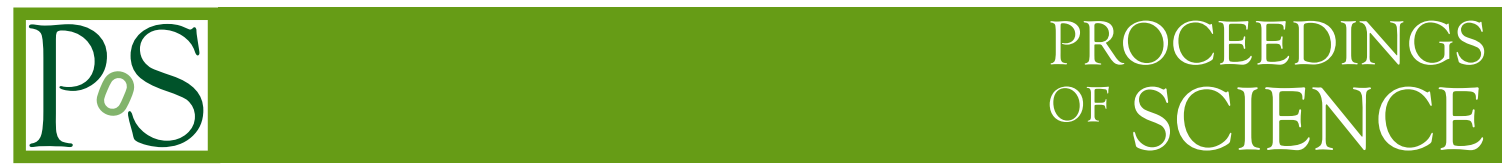

\title{
Top mass and width from CMS and ATLAS
}

\author{
Matteo Negrini ${ }^{\dagger}$,* \\ Istituto Nazionale di Fisica Nucleare - Sezione di Bologna, \\ Via Irnerio 46, 40126 Bologna, Italy \\ E-mail: matteo.negrini@cern.ch
}

An overview of recent measurements of the mass and width of the top quark is presented. The measurements are made by the CMS and ATLAS experiments, exploiting $p p$ collisions at the center-of-mass energies of $\sqrt{s}=8,13 \mathrm{TeV}$ in the Large Hadron Collider at CERN.

The Eighth Annual Conference on Large Hadron Collider Physics-LHCP2020 25-30 May, 2020

online

\footnotetext{
${ }^{*}$ Speaker

$\dagger$ On behalf of the ATLAS and CMS Collaborations
} 


\section{Introduction}

The top quark is the heaviest elementary particle in the Standard Model (SM). Its mass $\left(m_{t}\right)$ is a fundamental parameter of the SM: it allows to assess the internal consistency of the SM at the electroweak scale [1] and, assuming the validity of the SM up to very high scales, it enters in the determination of the lifetime of the universe [2].

The top quark, like all quarks, is not a free particle. Thus, its mass can be measured only through the comparisons of some observables with their theoretical expectations, in which $m_{t}$ is considered as a parameter of the theory. The current uncertainty on $m_{t}$ is at the sub-GeV level for single measurements and is approaching half- $\mathrm{GeV}$ in combinations.

Different measurement strategies are linked to different theoretical interpretations. In "direct" measurements, $m_{t}$ is obtained through the comparison of the experimental distributions of quantities that are sensitive to it (such as the invariant mass of the decay products of the top quark, but also other quantities can be used) and their theoretical expectations obtained from Monte Carlo (MC) simulation samples generated at different $m_{t}$ values. Direct techniques allow to measure the so-called MC mass $\left(m_{t}^{\mathrm{MC}}\right)$, that is the mass used as the MC simulation parameter. "Indirect" measurements are based on the comparison of a measured cross-section with its theoretical value that can be computed from first principle calculations. Indirect methods allow to determine the pole mass $\left(m_{t}^{\text {POLE }}\right)$ or the mass in the modified minimal subtraction $(\overline{\mathrm{MS}})$ renormalization scheme $\left(m_{t}^{\overline{\mathrm{MS}}}\right)$, depending on the theoretical scheme that is adopted in the calculation. The relation between $m_{t}^{\mathrm{POLE}}$ and $m_{t}^{\overline{\mathrm{MS}}}$ is calculated to 4-loop precision in QCD [3]; on the other hand the relation between $m_{t}^{\mathrm{MC}}$ and $m_{t}^{\mathrm{POLE}}$, believed to be at the level of a few hundreds of $\mathrm{MeV}$, is still under debate (see for example Refs. $[4,5]$ and references therein).

The theoretical top-quark width $\left(\Gamma_{t}\right)$, computed at the next-to-next-to-leading order (NNLO) in QCD within the $\mathrm{SM}$, is $\Gamma_{t}=1.322 \mathrm{GeV}$ [6]. "Indirect" techniques rely on the $\mathrm{SM}$ and on measurements of the branching fraction $B(t \rightarrow W b)$ to determine $\Gamma_{t}$ and allow to reach an uncertainty at the $100 \mathrm{MeV}$ level. "Direct" ones consist in the measurement of kinematic distributions that are sensitive to $\Gamma_{t}$. These are less precise, with a total uncertainty of few hundreds of $\mathrm{MeV}$, but have the advantage that the SM does not need to be assumed, therefore in principle they are sensitive to non-SM decays or couplings of the top quark that could emerge as deviations from the expected $\mathrm{SM} \Gamma_{t}$ value.

This paper presents an overview of some recent measurements of the mass and width of the top quark, obtained by the CMS [7] and ATLAS [8] experiments exploiting $p p$ collisions at the CERN Large Hadron Collider at the center-of-mass energies $\sqrt{s}=8,13 \mathrm{TeV}$.

\section{Top quark mass measurements}

The CMS collaboration measured $m_{t}^{\mathrm{MC}}$ using a simultaneous fit of the total $t \bar{t}$ production cross-section $\sigma_{t \bar{t}}$ and the $m_{t}^{\mathrm{MC}}$ parameter in the POWHEG simulation, using $35.9 \mathrm{fb}^{-1}$ of data at $\sqrt{s}=13 \mathrm{TeV}$ and selecting $t \bar{t}$ events, in which both $W$ bosons from $t \rightarrow W^{+} b$ decay leptonically ${ }^{1}$ (dilepton channel) [9]. $\sigma_{t \bar{t}}$ and $m_{t}^{\mathrm{MC}}$ are simultaneously determined from a fit, in which the sensitivity to $m_{t}^{\mathrm{MC}}$ is enhanced by including among the fitted observables the distribution of the

\footnotetext{
${ }^{1}$ Charge conjugated processes are implicitly included in this paper.
} 
minimum invariant mass of the combination of a charged lepton with a $b$-jet. The result is $m_{t}^{\mathrm{MC}}=172.33 \pm 0.14(\mathrm{stat})_{-0.72}^{+0.66}$ (syst) $\mathrm{GeV}$. The measured value of $\sigma_{t \bar{t}}$ is compared with theoretical calculations of the same quantities performed in the $\overline{\mathrm{MS}}$ and pole mass scheme to extract $m_{t}^{\overline{\mathrm{MS}}}$ and $m_{t}^{\mathrm{POLE}}$, respectively. The values depend on the PDF used in the calculation and have a larger total uncertainty than $m_{t}^{\mathrm{MC}}$, of the order of $\sim 2 \mathrm{GeV}$, slightly smaller for $m_{t}^{\overline{\mathrm{MS}}}$ than for $m_{t}^{\mathrm{POLE}}$ because of the better convergence of the $\overline{\mathrm{MS}}$ renormalization scheme in the calculation of $\sigma_{t \bar{t}}$.

The differential cross-section of the production of $t \bar{t}$ pairs in association with an energetic jet, as a function of the quantity $\rho_{s}=2 m_{0} / m_{t \bar{t}+1 \text { jet }}$, with $m_{0}$ constant and $m_{t \bar{t}+1 \text { jet }}$ the invariant mass of the $t \bar{t}+$ jet system, is highly sensitive to the top-quark mass value in the high $\rho_{s}$ region. The ATLAS collaboration exploited the availability of fixed-order calculation of the $t \bar{t}+1$ jet production process to measure both $m_{t}^{\overline{\mathrm{MS}}}$ and $m_{t}^{\mathrm{POLE}}$ using $20.2 \mathrm{fb}^{-1}$ of data at $\sqrt{s}=8 \mathrm{TeV}$ [10]. The value obtained for the pole mass scheme is $m_{t}^{\text {POLE }}=171.1 \pm 0.4$ (stat) $\pm 0.9(\text { syst })_{-0.3}^{+0.7}$ (theo) $\mathrm{GeV}$ while in the $\overline{\mathrm{MS}}$ scheme is $m_{t}^{\overline{\mathrm{MS}}}\left(m_{t}\right)=162.9 \pm 0.5$ (stat) $\pm 1.0(\text { syst })_{-1.2}^{+2.1}$ (theo) GeV.

Multi-differential cross-sections can also be used to determine $m_{t}$. Using $35.9 \mathrm{fb}^{-1}$ of data at $\sqrt{s}=13 \mathrm{TeV}$, the CMS collaboration measured $m_{t}^{\mathrm{POLE}}$ from the $t \bar{t}$ production triple-differential cross-section, with respect to the mass and rapidity of the $t \bar{t}$ system and the number of jets in the event [11]. In this work, the measured triple-differential cross-section is compared with nextto-leading order (NLO) predictions obtained with the aMC@NLO generator in fixed-order mode. $m_{t}^{\text {POLE }}$ is obtained by computing the $\chi^{2}$ values corresponding to predictions obtained using different $m_{t}^{\mathrm{POLE}}, \alpha_{S}\left(m_{Z}\right)$ and PDFs, and its value is $m_{t}^{\mathrm{POLE}}=170.5 \pm 0.7($ fit $) \pm 0.1(\text { model })_{-0.1}^{+0.0}($ param $) \pm$ 0.3 (scale) $\mathrm{GeV}$, in which the uncertainty is mainly due to "fit" uncertainty, determined with the criterion $\Delta \chi^{2}= \pm 1$.

Jets originated by $b$ quarks can be identified by the presence of a "soft" muon, from the semi-leptonic decay of a $b$-hadron, inside the jet. For $t \bar{t}$ events in which only one of the $t$ or $\bar{t}$ decays leptonically $(l+$ jets channel), that also contain one soft-muon tagged jet, it is possible to reconstruct the invariant mass of the pair of leptons (the soft one and the one from the leptonic top-quark decay), whose distribution is sensitive to the top-quark mass. This technique has the advantages of using a fully leptonic quantity that does not require the complete reconstruction of a top-quark, therefore reducing the uncertainty related to hadronic calibration and the modeling of the top-quark kinematics, as shown in the analysis of $36.1 \mathrm{fb}^{-1}$ of data at $\sqrt{s}=13 \mathrm{TeV}$ by the ATLAS collaboration [12]. The top-quark mass measured with this technique is $m_{t}^{\mathrm{MC}}=$ $174.48 \pm 0.40$ (stat) \pm 0.67 (syst) $\mathrm{GeV}$, in which the main contribution to the uncertainty is due to the modeling of the $b$ fragmentation and decay.

The CMS collaboration performed the first investigation of the running of the top-quark mass in the $\overline{\mathrm{MS}}$ scheme using $35.9 \mathrm{fb}^{-1}$ of data at $\sqrt{s}=13 \mathrm{TeV}$ [13]. In this analysis, a set of $t \bar{t}$ simulated events is divided in four sub-samples, corresponding to four intervals of the invariant mass of the $t \bar{t}$ pair determined at parton-level, which are treated as independent samples at the scales $\mu_{k}(k=1,4)$ defined as the average $m_{t \bar{t}}$ in each interval. A maximum likelihood unfolding is then applied to determine the true number of events at each scale from the observed one, which is needed to correct for reconstruction and resolution effects. For the determination of $m_{t}^{\overline{\mathrm{MS}}}$, measurements are compared with theoretical predictions at NLO in the $\overline{\mathrm{MS}}$ scheme as implemented in MCFM v6.8 [14]. The $m_{t}$ value at the $m_{t}$ scale $m_{t}\left(m_{t}\right)$ is determined independently in each 
$m_{t \bar{t}}$ interval, and the calculation of the corresponding $m_{t}\left(\mu_{k}\right)$ is done at one loop precision using CRunDec v3.0 [15]. The measured running mass is found to agree with the expected evolution from $m_{t}\left(m_{t}\right)=162.9 \pm 1.6\left(\text { fit }+ \text { extr }+\mathrm{PDF}+\alpha_{\mathrm{S}}\right)_{-3.0}^{+2.5}($ scale $) \mathrm{GeV}$.

The top-quark mass is strongly correlated to the invariant mass distribution of large-radius jets in highly boosted $t \bar{t}$ events. $m_{t}$ measurements obtained with these techniques, although affected by larger uncertainties, are becoming competitive with "standard" ones due to the recent improvement in the reconstruction techniques and calibration of large- $R$ jets. Highly boosted $t \bar{t}$ events in the $l+$ jets channel are selected in the analysis of $35.9 \mathrm{fb}^{-1}$ of data at $\sqrt{s}=13 \mathrm{TeV}$ by the CMS collaboration [16]. Top-quark candidates with $p_{\mathrm{T}}>400 \mathrm{GeV}$ are reconstructed with the XCone algorithm for two jets and using a radius parameter $R=1.2$; the same algorithm for three jets and with $R=0.4$ is applied to the hadronic top candidate constituents to reconstruct his sub-structure, and $m_{t}^{\mathrm{MC}}$ is then measured from the distribution of the invariant mass of the three sub-jets, unfolded at particle-level. The result $m_{t}^{\mathrm{MC}}=172.6 \pm 0.4$ (stat) \pm 1.6 (syst) \pm 1.5 (model) \pm 1.0 (theo) $\mathrm{GeV}$ shows an impressive improvement with respect to previous $m_{t}$ measurements using highly boosted top quarks, mainly due to the larger sample size and the reconstruction algorithm adopted.

\section{Top-quark width measurements}

The most precise measurement of the decay width of the top-quark is $\Gamma_{t}=1.36 \pm 0.02$ (stat) ${ }_{-0.11}^{+0.14}$ (scale) $\mathrm{GeV}$ and was obtained by the CMS collaboration from a measurement of the branching fraction $B(t \rightarrow W b)$, assuming the validity of the SM [17].

Recently, the ATLAS collaboration performed a direct measurement of $\Gamma_{t}$ using $139 \mathrm{fb}^{-1}$ of data at $\sqrt{s}=13 \mathrm{TeV}$, and reconstructing $t \bar{t}$ events in the di-leptonic channel [18]. The invariant mass distribution of the lepton and $b$-jet system, associated to the same top quark candidate, is sensitive to $\Gamma_{t}$. The analysis strategy consists in the creation of templates corresponding to different $\Gamma_{t}$ values, that are used in profile likelihood fit on data. The measured top-quark width $\Gamma_{t}=1.94_{-0.49}^{+0.52} \mathrm{GeV}$, in which the main contributions to the uncertainty are due to jet calibration and signal and background modeling, is in good agreement with the SM expectation.

\section{Summary}

The top-quark mass is a fundamental parameter of the SM, which allows precision tests of the SM and provides insights on the fate of the universe. Improving on the current uncertainty of few hundred $\mathrm{MeV}$ poses experimental and theoretical challenges, therefore the ultimate $m_{t}$ determination does not require a single measurement but a physics program that includes: techniques with uncertainties coming from different sources and the possibility to combine the measurements, measurements with different theoretical interpretations.

Direct measurements of $\Gamma_{t}$ are in principle sensitive to non-SM decays or couplings of the top quark, however they are consistent with SM expectations within the present uncertainty.

\section{References}

[1] M. Baak et al. [Gfitter Group], The global electroweak fit at NNLO and prospects for the LHC and ILC, Eur. Phys. J. C 74 (2014), 3046 [arXiv:1407.3792 [hep-ph]]. 
[2] G. Degrassi, S. Di Vita, J. Elias-Miro, J. R. Espinosa, G. F. Giudice, G. Isidori and A. Strumia, Higgs mass and vacuum stability in the Standard Model at NNLO, JHEP 08 (2012), 098 [arXiv:1205.6497 [hep-ph]].

[3] P. Marquard, A. V. Smirnov, V. A. Smirnov and M. Steinhauser, Quark Mass Relations to FourLoop Order in Perturbative QCD, Phys. Rev. Lett. 114 (2015), 142002 [arXiv:1502.01030 [hep-ph]].

[4] P. Nason, The Top Mass in Hadronic Collisions, [arXiv:1712.02796 [hep-ph]].

[5] A. H. Hoang, What is the Top Quark Mass?, Ann. Rev. of Nucl. and Part. Sci. 70 (2020), [arXiv:2004.12915 [hep-ph]].

[6] J. Gao, C. S. Li and H. X. Zhu, Top Quark Decay at Next-to-Next-to Leading Order in QCD, Phys. Rev. Lett. 110 (2013), 042001 [arXiv:1210.2808 [hep-ph]].

[7] CMS Collaboration, The CMS experiment at the CERN LHC, 2008 JINST 3 S08004.

[8] ATLAS Collaboration, The ATLAS Experiment at the CERN Large Hadron Collider, 2008 JINST 3 S08003.

[9] CMS Collaboration, Measurement of the tit production cross section, the top quark mass, and the strong coupling constant using dilepton events in pp collisions at $\sqrt{s}=13 \mathrm{TeV}$, Eur. Phys. J. C 79 (2019), 368 [arXiv:1812.10505 [hep-ex]].

[10] ATLAS Collaboration, Measurement of the top-quark mass in $t \bar{t}+1$-jet events collected with the ATLAS detector in pp collisions at $\sqrt{s}=8 \mathrm{TeV}$, JHEP 11 (2019), 150 [arXiv:1905.02302 [hep-ex]].

[11] CMS Collaboration, Measurement of $\overline{\mathrm{t}}$ normalised multi-differential cross sections in $p p$ collisions at $\sqrt{s}=13 \mathrm{TeV}$, and simultaneous determination of the strong coupling strength, top quark pole mass, and parton distribution functions, [arXiv:1904.05237 [hep-ex]].

[12] ATLAS Collaboration, Measurement of the top quark mass using a leptonic invariant mass in pp collisions at $\sqrt{s}=13 \mathrm{TeV}$ with the ATLAS detector, ATLAS-CONF-2019-046, https://cds.cern.ch/record/2693954.

[13] CMS Collaboration, Running of the top quark mass from proton-proton collisions at $\sqrt{s}=13$ TeV, Phys. Lett. B 803 (2020), 135263 [arXiv:1909.09193 [hep-ex]].

[14] J. M. Campbell and R. K. Ellis, MCFM for the Tevatron and the LHC, Nucl. Phys. B Proc. Suppl. 205-206 (2010), 10 [arXiv:1007.3492 [hep-ph]].

[15] B. Schmidt and M. Steinhauser, CRunDec: a C++ package for running and decoupling of the strong coupling and quark masses, Comput. Phys. Commun. 183 (2012), 1845 [arXiv:1201.6149 [hep-ph]]. 
[16] CMS Collaboration, Measurement of the Jet Mass Distribution and Top Quark Mass in Hadronic Decays of Boosted Top Quarks in pp Collisions at $\sqrt{s}=13 \mathrm{TeV}$, Phys. Rev. Lett. 124 (2020), 202001 [arXiv:1911.03800 [hep-ex]].

[17] CMS Collaboration, Measurement of the ratio $\mathcal{B}(t \rightarrow W b) / \mathcal{B}(t \rightarrow W q)$ in pp collisions at $\sqrt{s}=8 \mathrm{TeV}$, Phys. Lett. B 736 (2014), 33 [arXiv:1404.2292 [hep-ex]].

[18] ATLAS Collaboration, Measurement of the top-quark decay width in top-quark pair events in the dilepton channel at $\sqrt{s}=13 \mathrm{TeV}$ with the ATLAS detector, ATLAS-CONF-2019-038, https://cds.cern.ch/record/2684952. 